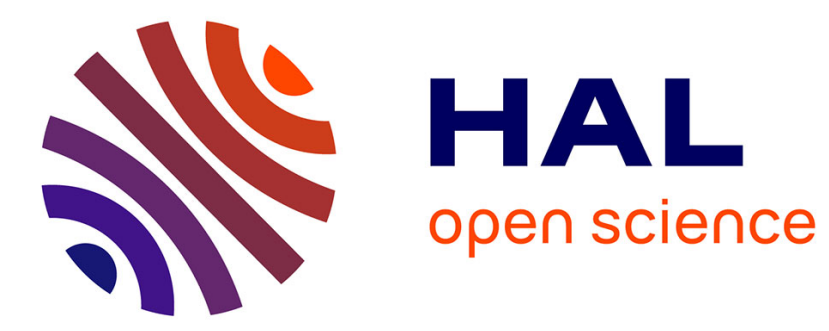

\title{
Accurate phase-shifting digital interferometry
}

Michael Atlan, Michel Gross, Emilie Absil

\section{To cite this version:}

Michael Atlan, Michel Gross, Emilie Absil. Accurate phase-shifting digital interferometry. Optics Letters, 2007, 32 (11), pp.1456. hal-00265964

\section{HAL Id: hal-00265964 https://hal.science/hal-00265964}

Submitted on 20 Mar 2008

HAL is a multi-disciplinary open access archive for the deposit and dissemination of scientific research documents, whether they are published or not. The documents may come from teaching and research institutions in France or abroad, or from public or private research centers.
L'archive ouverte pluridisciplinaire HAL, est destinée au dépôt et à la diffusion de documents scientifiques de niveau recherche, publiés ou non, émanant des établissements d'enseignement et de recherche français ou étrangers, des laboratoires publics ou privés. 


\title{
Accurate phase-shifting digital interferometry
}

\author{
M. Atlan and M. Gross \\ École Normale Supérieure, \\ Laboratoire Kastler-Brossel, UMR 8552 \\ 24 rue Lhomond 75231 Paris cedex 05. France
}

E. Absil

École Supérieure de Physique et de Chimie Industrielles de la Ville de Paris, Laboratoire d'Optique, CNRS UPR A0005, Université Pierre et Marie Curie, 10 rue Vauquelin 75231 Paris cedex 05. France

(Dated: March 20, 2008)

In phase-shifting interferometry experiments, the accuracy of the phase shift is a major issue. A lot of experimental and data analysis effort are done to cancel phase shift errors inherent to the modulation techniques used. We propose here to remove most of the phase shift error by having recourse to a electronic frequency shift method. This approach can be applied to both holography and interferometry. We validate the idea with an holographic experiment.

Phase shifting interferometry [1] and holography [2] are powerful tools which have been in use for many years. In such systems, the phase of the reference arm of an interferometer is shifted by a mirror mounted on a piezoelectric transducer (PZT) and the interference pattern is recorded by a CCD camera. Since the PZT is not perfectly linear, nor calibrated, phase shift errors are introduced [3]. This yields measurement errors and twin image aliases [4]. To suppress or compensate these errors many techniques and algorithms have been proposed [516]. Some of these techniques [17] yield discussion and controversy $[18,19]$.

Here, we propose to suppress most of the phase error by replacing the phase shift $\Delta \varphi$ by a frequency shift $\Delta \omega$ induced by Bragg cells (Acousto Optic modulator) driven by RF (Radio Frequency) signals. We will show that the phase, which is driven by sine wave electronic signals, is very accurate. This technique has been introduced in the past in the context of holography [20, 21]), but it can be adapted to interferometry. In this letter, we will describe the technique, and evaluate the phase shift errors by measuring the relative weight of the twin image component in an off-axis Mach-Zehnder configuration.

The setup is shown on Fig.1. It is similar to the one used in [20]. The main laser beam (complex field $E_{L}$, frequency $\omega_{L}$ ) is provided by a Sanyo DL-7140-201 diode laser $(\lambda=780 \mathrm{~nm}, 50 \mathrm{~mW}$ for $95 \mathrm{~mA}$ of current). It is split into an illumination $\left(E_{I}, \omega_{I}\right)$ and a local oscillator (LO) beam $\left(E_{L O}, \omega_{L O}\right)$. A set of optical attenuators A1, A2 (grey neutral filters) allows to adjust the illumination and the LO beam intensities. The object is an USAF target in transmission (back illuminated). The CCD camera (PCO Pixelfly digital camera: $12 \mathrm{bit}$, frame rate $\omega_{C C D} \simeq 10 \mathrm{~Hz}$, acquisition time $T_{C C D}=1 / \omega_{C C D} \simeq 100 \mathrm{~ms}$, with $1280 \times 1024$ pixels of $6.7 \times 6.7 \mu \mathrm{m})$ records the interference pattern of the object field $E$ (whose frequency $\omega$ is unshifted with respect to illumination: $\omega=\omega_{I}$ ) with the LO field $E_{L O}$. A set of two acousto optic modulators AOM1 and AOM2 (Crystal Technology: $\omega_{A O M 1,2} \simeq 80 \mathrm{MHz}$ ) shift the frequency of

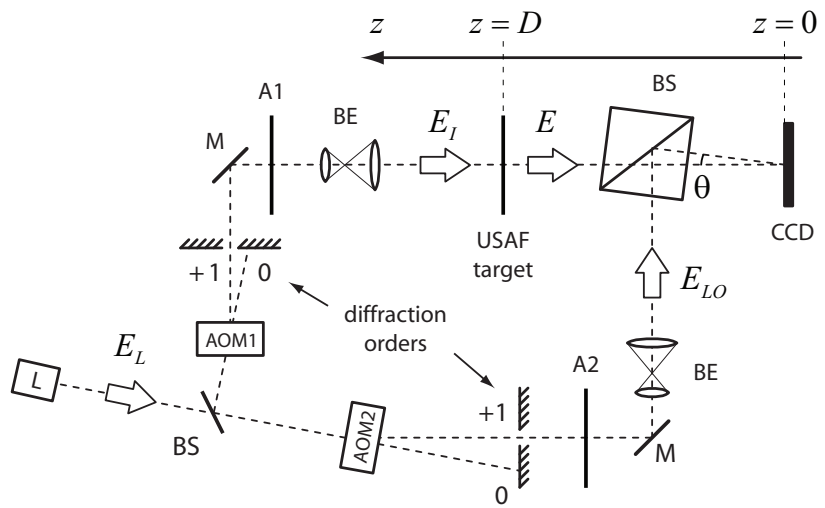

FIG. 1: phase-shifting digital holography setup. L: main laser; BS: Beam splitter; AOM1 and AOM2: acousto-optic modulators; BE: beam expander; M: mirror; A1/2: light attenuator. USAF: transmission USAF 1951 test pattern. CCD : CCD camera. $E_{L}, E_{I}, E_{L O}$ and $E$ : laser, illumination, LO and object fields.

the illumination and LO beams $\left(\omega_{I}=\omega=\omega_{L}+\omega_{A O M 1}\right.$ and $\left.\omega_{L O}=\omega_{L}+\omega_{A O M 2}\right)$, so that the heterodyne beat frequency can be freely adjusted:

$$
\Delta \omega \equiv \omega_{L O}-\omega_{I}=\omega_{A O M 1}-\omega_{A O M 2}
$$

The relative phase shift $\Delta \varphi$ of 2 consecutive images is:

$$
\Delta \varphi(\Delta \omega)=2 \pi \Delta \omega / \omega_{C C D}
$$

In the following, we will measure the signal field $E$ by using the 4 phases method: $\Delta \varphi=m \pi / 2$ where $m$ is an integer. The complex signal field amplitude $E$ is then obtained from a sequence $I_{0}, I_{1} \ldots I_{3}$ of 4 consecutive CCD intensity images by [20]:

$$
E=A \sum_{m=0}^{3}(j)^{m} I_{m}=A\left[\left(I_{0}-I_{2}\right) \pm j\left(I_{1}-I_{3}\right)\right]
$$



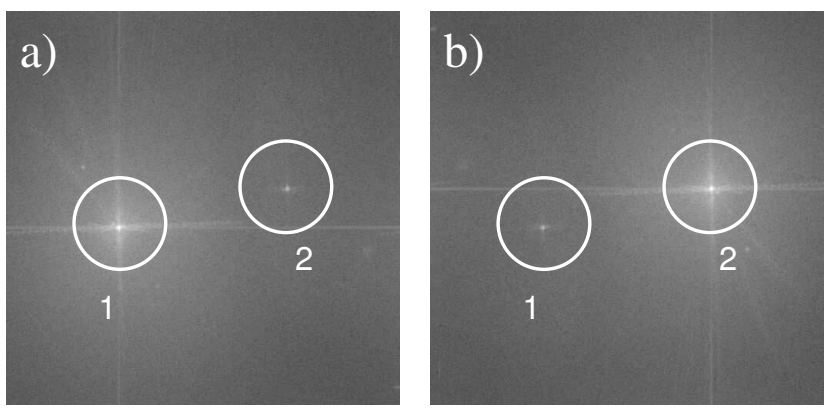

FIG. 2: USAF target k-space $1024 \times 1024$ holograms $\tilde{E}\left(k_{x}, k_{y}\right)$ displayed in logarithmic scale for $\left|\tilde{E}\left(k_{x}, k_{y}\right)\right|^{2}$ at $\Delta \omega=+2.5$ $\mathrm{Hz}$ (a) and $\Delta \omega=-2.5 \mathrm{~Hz}$ (b). The center of the white circles correspond to the true (1) and twin (2) images.

where $A$ is a normalization factor which depends on $E_{L O}$, and $j^{2}=-1$.

In comparison to a standard phase-shifting experiment, we have replaced the phase shifts $\Delta \varphi$ of the LO induced by the displacement of a mirror mounted on a PZT, by a frequency shift $\Delta \omega$ generated through the dynamic Bragg gratings. The AOMs are driven by two numeric synthesizers (Agilent 33250A), which are in coherence, the first synthesizer (master) providing the $10 \mathrm{MHz}$ reference of the second synthesizer (slave). Since the dynamic phase shift we apply is accurately controlled, the twin image alias [22] can be cancelled efficiently. To verify this point quantitatively, we have measured the field $E$ diffracted by the USAF target, for different values of the heterodyne beat frequency $\Delta \omega$. A map of the field $E$ is computed from a sequence of 4 recorded frames, according to Eq.3. To separate the "true" image signal from the "twin" image alias, the USAF target is angularly shifted (angle $\theta \sim 1^{\circ}$ ) with respect to the LO beam direction. From the field $E(x, y)$ where $x, y$ are the CCD pixels coordinates, we calculate by 2D (fast) Fourier transformation $(F T 2 D)$ the k-space field $\tilde{E}\left(k_{x}, k_{y}\right)=F T 2 D E(x, y)$, in which the "true" and "twin" images are well separated when the object is off-axis.

Fig.2 shows the k-space field intensity distribution $\left(\left|\tilde{E}\left(k_{x}, k_{y}\right)\right|^{2}\right)$ in logarithmic scale for $\Delta \omega=+2.5 \mathrm{~Hz}$ (a) and $-2.5 \mathrm{~Hz}(\mathrm{~b})$. Here, the $\mathrm{k}$-space field is computed by Fast Fourier Transform (FFT) from the $1280 \times 1024$ CCD data that are truncated into a $1024 \times 1024$ calculation grid. Since the illumination beam is a plane wave, and since the USAF target transmits a large fraction of the illumination beam, the object field $E$ remains roughly a plane wave. Thus, the USAF true image corresponds, in $\mathrm{k}$-space, to a narrow bright spot visible in the center of the white circle (region 1) in Fig.2a. The twin image (center of circle 2) is the symmetric of true image with respect to the center of the k-space. On Fig.2a, with $\Delta \omega=+2.5 \mathrm{~Hz}$, the heterodyne detection allows one to select the true image, and reject the twin image. The true image is bright, and the twin image is dark. On

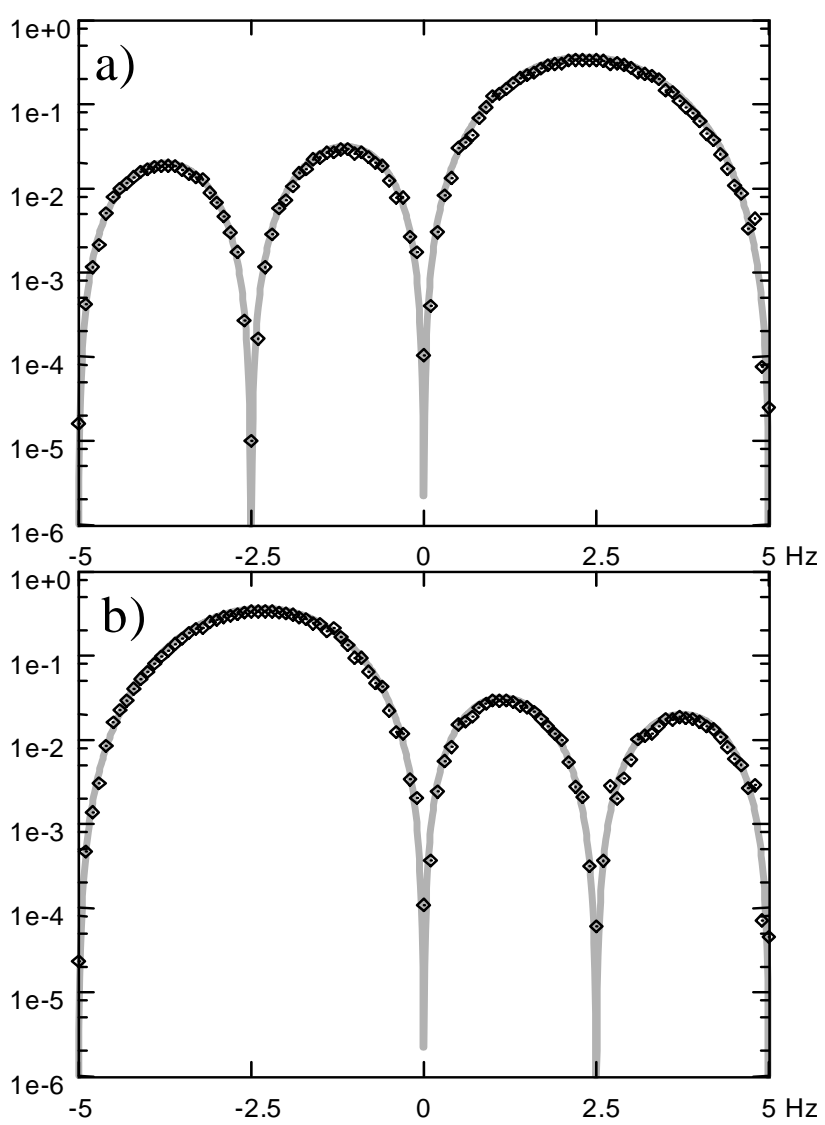

FIG. 3: a) Weight $W_{+1}$ of the true image peak as a function of the heterodyne frequency $\Delta \omega$ in Hz. Vertical axis is in logarithmic scale arbitrary units. The points correspond to experimental data: $\Delta \omega=-5$ to $+5 \mathrm{~Hz}$ with $0.1 \mathrm{~Hz}$ increment. The solid grey line is the theory (Eq.5) . b) Weight $W_{-1}$ of the twin image.

Fig.2b, on the contrary, $\Delta \omega=-2.5 \mathrm{~Hz}$ so that the true image (1) is dark, while the twin image (2) is bright.

We have measured the weight of the true $\left(W_{+1}\right)$ and twin $\left(W_{-1}\right)$ image peak defined by:

$$
W_{ \pm 1}=\sum_{C_{ \pm 1}}\left|\tilde{E}_{k_{x}, k_{y}}\right|^{2}
$$

where $\sum_{C_{ \pm 1}}$ is the sum over a $10 \times 10$ pixels region centered on the true image $\left(C_{+1}\right)$ and twin image peak $\left(C_{-1}\right)$ respectively. By sweeping the AOM1,2 synthesizer frequency, we have studied how $W_{ \pm 1}$ vary with the heterodyne frequency $\Delta \omega$.

Fig.3 shows $W_{ \pm 1}$ as a function of $\Delta \omega$. When the 4phases condition is fulfilled, i.e. when $\Delta \omega=+2.5 \mathrm{~Hz}$, the weight of true image is maximum, $\left(W_{+1} \simeq 0.3\right)$ while the twin image is nearly zero $\left(W_{-1} \simeq 10^{-4}\right)$. Contrarily, when $\Delta \omega=-2.5 \mathrm{~Hz}, W_{+1}$ is $\simeq 10^{-5}$ while $W_{-1} \simeq 0.3$ ). The relative weight of the parasitic component is thus $W_{+1} / W_{-1} \simeq 3 \times 10^{-4}$ when one selects the true image $(+2.5 \mathrm{~Hz})$, and $W_{-1} / W_{+1} \simeq 3 \times 10^{-5}$ when one selects 

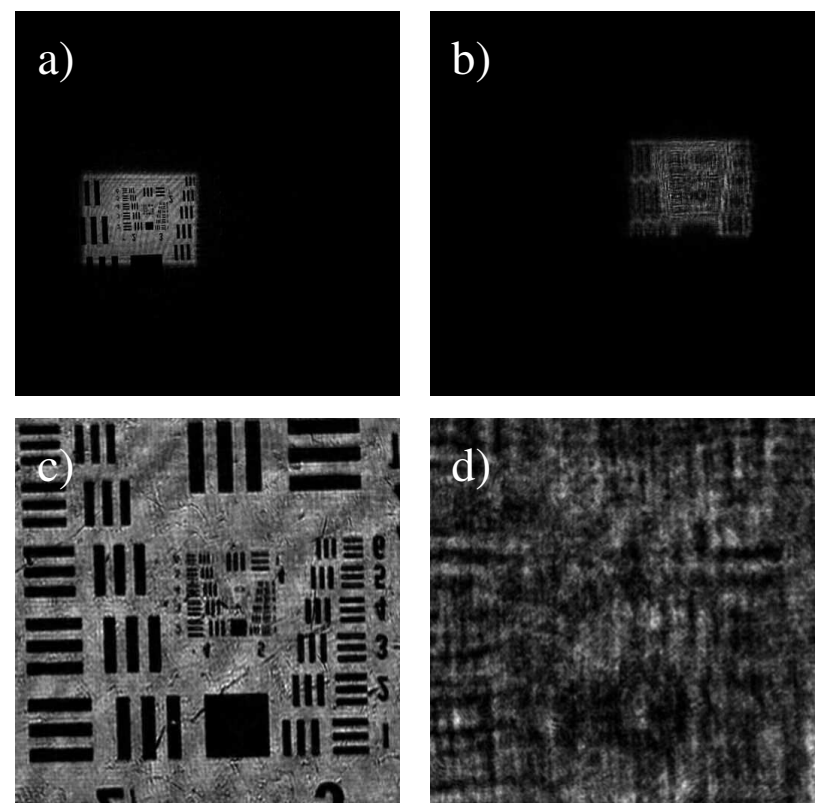

FIG. 4: a,b) USAF target reconstructed image in linear scale for the field intensity $\left|E_{x, y, z}\right|^{2}$ with $z=194.5 \mathrm{~mm}(4096 \times 4096$ pixels): a) $\Delta \omega=+2.5 \mathrm{~Hz}$ (true image); b) $\Delta \omega=-2.5 \mathrm{~Hz}$ (twin image). c) Zoom of the center of the true image a $(512 \times 512$ pixels $)$. d) Zoom of the center of the twin image b: $512 \times 512$ pixels. Pixel size is $6.7 \times 6.7 \mu \mathrm{m}$.

the twin image $(-2.5 \mathrm{~Hz})$. The parasitic twin image component can thus be neglected. We have computed the theoretical shape of $W_{ \pm 1}$ (solid gray lines). Since the $E . E_{L O}^{*}$ heterodyne term is simply proportional to $e^{j m \Delta \varphi}$ where $m$ is the index of the image $I_{m}$ within the sequence. We get from Eq.3:

$$
W_{ \pm 1}(\Delta \omega)=B \eta^{2}(\Delta \omega)\left|\sum_{m=0}^{3} j^{m} e^{\mp j m \Delta \varphi(\Delta \omega)}\right|^{2}
$$

Here $B$ is a proportionality factor and $\Delta \varphi(\Delta \omega)$ is given by Eq.2. $\eta$ is the factor that describes the reduction of the detection efficiency $(0<\eta<1)$, because of the phase drift during the acquisition time of an image (i.e. $\left.T_{C C D}=1 / \omega_{C C D}\right)$. Since the phase drift is constant in time, this efficiency factor takes the form :

$$
\eta(\Delta \omega)=\frac{\left|\int_{\varphi=0}^{\Delta \varphi(\Delta \omega)} e^{j \varphi} d \varphi\right|}{\Delta \varphi(\Delta \omega)}=\frac{\left|1-e^{j \Delta \varphi(\Delta \omega)}\right|}{\Delta \varphi(\Delta \omega)}
$$

As depicted in Fig.3, the experimental results for $W_{ \pm 1}(\Delta \omega)$ (points) agree with Eq.5 (solid grey lines).

To illustrate the ability to reconstruct an image and to cancel the twin image alias, we have calculated the image of the USAF target from the complex field $E(x, y)$ (i.e. from the complex hologram recorded in the CCD plane). The image is reconstructed by the standard convolution method $[22,23]$ that yields a calculation grid equal to the pixel size [24]. To calculate the convolution product, we have used the Fourier method, like in [20]. To obtain a proper off-axis image, we have enlarged the $1280 \times 1024$ calculation grid that corresponds to the dimensions of the CCD by padding the data into a $4096 \times 4096$ null matrix $[25,26]$ (zero padding method).

Fig.4a shows the USAF image obtained for a reconstruction distance $z=194.5 \mathrm{~mm}$; it corresponds to the true image revealed at the modulation frequency $\Delta \omega=+2.5 \mathrm{~Hz}$. We can notice that the zero order and twin image aliases are not visible. Fig. $4 \mathrm{~b}$ shows the twin image obtained at $\Delta \omega=-2.5 \mathrm{~Hz}$. With the chosen reconstruction distance $z=194.5 \mathrm{~mm}$, the twin image is blurred. The true image and zero-order contributions are not visible. If the image reconstruction is made with $z=-194.5 \mathrm{~mm}$, the reconstructed twin image becomes identical to the Fig.4a true image. Fig.4c is a zoomed part of Fig.4a true image. Fig.4d is a zoomed part of Fig. $4 \mathrm{~b}$ twin image.

In conclusion, we have proposed to replace, in phaseshifting interferometry (or holography), the PZT driven mechanical motion of an optical component (mirror), by AOMs driven by numeric synthesizers. The mechanical phase shift is thus replaced by an electronic frequency shift, which is much more accurate. Most of the phase errors are thus suppressed by this technique, which is simple to manage. This result is confirmed by experiment. The proposed technique is expected to greatly simplify data analysis, improving the measurement accuracy.

The authors acknowledge support from the French National Research Agency (ANR).
[1] K. Creath. "Phase measurement interferometry techniques" in Progress in Optics, volume 26. E.Wolf, ed. (Elsevier, 1988), 1988.

[2] I. Yamaguchi and T. Zhang. Phase-shifting digital holography. Optics Letters, 18(1):31, 1997.

[3] K. Kinnstaetter, A. W. Lohmann, J. Schwider, and N. Streibl. Accuracy of phase shifting interferometry. Appl. Opt. , 27:5082-5089, December 1988.

[4] Etienne Cuche, Pierre Marquet, and Christian Depeursinge. Spatial filtering for zero-order and twin-image elimination in digital off-axis holography. Appl. Opt., 39(23):4070-4075, 10 August 2000.

[5] J. Schwider, R. Burow, K.-E. Elssner, J. Grzanna, R. Spolaczyk, and K. Merkel. Digital wave-front measuring interferometry: some systematic error sources. Appl. Opt., 22:3421-3432, 1983.

[6] P. Hariharan, B. F. Oreb, and T. Eiju. Digital phaseshifting interferometry: a simple error-compensating phase calculation algorithm. Appl. Opt., 26:2504-2505, 1987. 
[7] G. Lai and T. Yatagai. Generalized phase-shifting interferometry. J. Opt. Soc. Am. A, 8:822-827, 1991.

[8] K. G. Larkin and B. F. Oreb. Design and assessment of symmetrical phase-shifting algorithms. J. Opt. Soc. Am. A, 9:1740-1748, 1992 .

[9] P. L. Taylor G. D. Lassahn, J. K. Lassahn and V. A. Deason. Multiple fringe analysis with unknown phase shifts. Opt. Eng., 33:2039-2044, 1994.

[10] J. Schmit and K. Creath. Extended averaging technique for derivation of error-compensating algorithms in phaseshifting interferometry. Appl. Opt., 34:3610-3619, 1995.

[11] B. Gutman and H. Weber. Phase-shifter calibration and error detection in phase-shifting applications: a new method. Appl. Opt., 37:7624-7631, 1998.

[12] J. M. Huntley. Suppression of phase errors from vibration in phase-shifting interferometry. J. Opt. Soc. Am. A, 15:2233-2241, 1998.

[13] C. S. Guo, L. Zhang, H. T. Wang, J. Liao, and Y. Y. Zhu. Phase-shifting error and its elimination in phase-shifting digital holography. Opt. Lett., 27:1687-1689, 2002.

[14] L. Z. Cai, Q. Liu, and X. L. Yang. Generalized phaseshifting interferometry with arbitrary unknown phase steps for diffraction objects. Opt. Lett., 29:183-185, 2004.

[15] L. Z. Cai, Y. R. Wang Q. Liu, X. F. Meng, and M. Z. He. Experimental demonstrations of the digital correction of complex wave errors caused by arbitrary phaseshift errors in phase-shifting interferometry. Appl. Opt., 45:1193-1202, 2006.

[16] X. F. Xu, L. Z. Cai, X. F. Meng, G. Y. Dong, and X. X. Shen. Fast blind extraction of arbitrary unknown phase shifts by an iterative tangent approach in generalized phase-shifting interferometry. Opt. Lett., 31:1966-1968, 2006 .
[17] L. Z. Cai, Q. Liu, and X. L. Yang. Phase-shift extraction and wave-front reconstruction in phase-shifting interferometry with arbitrary phase steps. Opt. Lett., 28:1808$1810,2003$.

[18] J. A. Ferrari and E. Garbusi. Comment on "phaseshift extraction and wave-front reconstruction in phaseshifting interferometry with arbitrary phase steps". Opt. Lett., 29:1257-1258, 2004.

[19] L. Z. Cai, Q. Liu, and X. L. Yang. Reply to comment on "phase-shift extraction and wave-front reconstruction in phase-shifting interferometry with arbitrary phase steps". Optics Letters, 29(11):1259-1260, 2004.

[20] F. LeClerc, L. Collot, and M. Gross. Numerical heterodyne holography using $2 \mathrm{~d}$ photo-detector arrays. Optics Letters, 25:716, Mai 2000.

[21] F. LeClerc, L. Collot, and M. Gross. Synthetic-aperture experiment in visible with on-axis digital heterodyne holography. Optics Letters, 26, October 2001.

$[22]$ U. Schnars. Direct phase determination in hologram interferometry with use of digitally recorded holograms. JOSA A., 11:977, July 1994.

[23] T. Kreis. Digital holography for metrologic applications. in Interferometry in Speckle Light, P. Jacquot and J.-M. Fournier, eds., Springer Verlag, pages 205-212, 2000.

[24] F. Zhang, I. Yamaguchi, and L. P. Yaroslavsky. Algorithm for reconstruction of digital holograms with adjustable magnification. Opt. Lett., 29:1668-1670, 2004.

[25] T. Kreis, M. Adams, and W. Jptner. Methods of digital holography: a comparison. Proc. SPIE, 3098, 1997.

[26] I. Yamaguchi, J. i. Kato, S. Ohta, and J. Mizuno. Image formation in phase-shifting digital holography and applications to microscopy. Appl. Opt., 40(16):6177-6186, 2001. 